nosis, management and surgical techniques, while also dealing with complications and outcomes. Despite its compact size, it contains over 980 index references, with detailed subsections including rheumatoid arthritis (19 subindexed topics), flaps (over 30) and the wrist (nearly 40).

Physiotherapy regimens, eponyms and classifications are fully and concisely presented in an easily accessible format so that its 18 chapters also serve as a unique, singlevolume source for examination revision, though it might have been useful in that regard to have included key references. An introductory anatomy summary would also have been helpful. And it would be churlish to notice that 'metephyseal', and 'trapezioid' have sneaked past the subeditor.

This excellent volume delivers crucial information and advice, elegantly presented in a practical and trim format. Its 345 grams sit easily in the pocket of a white coat and to adapt a metaphor to recognise the importance of hand function, it punches well above its weight.

\section{Management of Cleft Lip and Palate in the Developing World}

MICHAEL MARS, DEBBIE SELL, ALEX HABE

\begin{tabular}{ll}
\hline EXTENT P/H & 221 pages, Paperback \\
PRICE/ISBN & $£ 34.99,9780470019689$ \\
PUBLISHER & Wiley (Chichester), 2008 \\
REVIEWER & Michael Earley \\
STAR RATING & $* * * *$
\end{tabular}

This is a practical account of what is being done in an often controversial area giving a balanced view regarding motivations, training and sustainability. The introduction by the three editors puts the case very well for treating cleft lip and palate in the developing world by non-governmental organisations (NGOs).

It is divided into five parts: Surgery and Anaesthesia; Approaches to organisation; Facial growth research; Medical Management, disability, psychological and social aspects; and Speech and Audiology.

Part one is an excellent exposition of the problems faced by large NGOs acting for altruistic reasons. As in any multiauthored book. there is a risk of repetition and unfortunately this is no exception. However, this gives a certain advantage, allowing each chapter to stand alone for the reader. Chapter 4 presents a unique opportunity to read one surgeon's rather entrenched views. It unfortunately detracts from the more balanced efforts made elsewhere throughout the book. Chapter 5 on anaesthesia would probably sit better if chapter 4 were excluded.

Part two gives an invaluable insight into how large organi- sations such as Operation Smile and Rotaplast managed to grapple with growth, governance and management. The chapter on logistics and nursing issues reads like a how to do it properly' manual and the final chapter on the Sri Lankan project shows how it was all put together to achieve service, teaching, research and sustainability in one site. Part three on facial growth would probably be better as an appendix as it interrupts the general flow of the book.

Part four raises the larger questions beyond those relating to management of cleft lip and palate alone and discusses general health, cultural and psychological issues. Each chapter could easily stand alone and each makes fascinating reading. The most important outcome measurement for palate repair is speech and it is a pity that the excellent chapters on speech and language come at the end.

In conclusion, this is a unique book that is attempting something never done before. In spite of its slightly disordered presentation, it is essential reading for anyone interested in volunteer work overseas.

\section{Surgical Pathology of Endocrine and Neuroendocrine Tumors}

ASHRAF KHAN

$\begin{array}{ll}\text { EXTENT P/H } & 254 \text { pages, Hardback } \\ \text { PRICE/ISBN } & £ 99.00,9781603273954 \\ \text { PUBLISHER } & \text { Humana (New York), } 2009 \\ \text { REVIEWER } & \text { Adrian Bateman } \\ \text { STAR RATING } & * * * *\end{array}$

This book contains a comprehensive review of neoplasms of the endocrine system and of neuroendocrine tumours arising at other sites. The book has multiple authors who are recognised experts in their field. The book starts with an interesting section on imaging of these tumours followed by two chapters on fine needle aspiration cytology. Tumours (endocrine and nonendocrine) of the pituitary, thyroid, parathyroid and adrenal glands are then discussed, followed by chapters on endocrine tumours arising within the respiratory system, gastrointestinal tract and pancreas, breast, urogenital tract, thymus and skin.

The final chapters discuss endocrine tumours arising in younger patients, inherited syndromes and the application of molecular diagnostic techniques. The latter techniques are becoming increasingly integrated with - and complementary to - morphological assessment in diagnostic cellular pathology and it is, therefore, very important that this topic has been included. The book is well illustrated and each chapter is extensively referenced. The indexing system appears appropriate.

This book would be particularly suitable as a reference text for a general histopathologist or a specialist histopathologist practising in endocrine pathology. However, it would also be of 
interest to specialists reporting in one of the other organ systems in which endocrine tumours arise.

The terminology of endocrine/neuroendocrine neoplasms arising outside the usual endocrine organs can be varied and at times confusing. For example, the terms 'carcinoid' and 'atypical carcinoid' are still in common use for tumours arising within the lung but the term '(neuro)endocrine tumour' is now recommended for neoplasms that were previously called 'carcinoid tumour' within the gastrointestinal tract. Where relevant, this issue is carefully discussed within individual chapters.

Overall, this is a very well written and carefully illustrated book that I would recommend to histopathologists practising in anatomical areas in which endocrine tumours are encountered.

\section{Basic Surgical Techniques, 6th edn}

RM KIRK

$\begin{array}{ll}\text { EXTENT P/H } & 206 \text { pages, Paperback } \\ \text { PRICE/ISBN } & £ 36.99,9780702033919 \\ \text { PUBLISHER } & \text { Churchill Livingstone (London), } 2010 \\ \text { REVIEWER } & \text { Robert Whistance } \\ \text { STAR RATING } & * * * * *\end{array}$

This comprehensive, yet concise, book describes the basic surgical techniques required of junior trainees across the breadth of surgical specialties. It consists of 14 chapters, beginning with theatre conduct, instrument handling and suturing, before providing detailed descriptions of the elementary skills required to handle blood vessels, bowel and bone. It finishes by offering advice on preventing and managing unwanted complications such as bleeding and infection.

The author's formal style is clear yet entertaining, while the content is contemporary with modern surgical practice. The author also delves into some of the basic techniques required for each of the surgical specialties, expanding the audience for which the book is appropriate and making it an ideal accompanying text for students undertaking the Basic Surgical Skills course. The book also bridges the gap between surgical pathology texts and true operative scripts and is ideal for medical students, foundation doctors and core surgical trainees. I certainly would have benefited from reading this text prior to beginning my surgical training. The prose is supplemented with frequent illustrations that assist in the understanding of the complex practical procedures described. All images are in colour and provide a step-by-step pictorial guide to each of the surgical techniques.

Overall, this is an exceptionally well-written book that appropriately meets the needs of its target audience in a concise manner. I would recommend this book to surgical trainees and it has certainly met the author's aims.

\section{Paediatric Orthopaedics: A System of Decision-Making}

BENJAMIN JOSEPH, SELVADURAI NAYAGAM, RANDALL T LODER, IAN TORODE

\begin{tabular}{ll}
\hline EXTENT P/H & 527 pages, Hardback \\
PRICE/ISBN & $£ 99.00,9780340889459$ \\
PUBLISHER & Hodder Arnold (London), 2009 \\
REVIEWER & Nick Clarke \\
STAR RATING & $* * * * *$
\end{tabular}

Paediatric orthopaedics as a speciality is in a time of transition. There is a focus on subspecialisation within paediatrics but, at the same time, there are proponents of site-specific involvement by adult regional surgeons, for example, knee reconstruction. This important text is a timely reminder that the management of paediatric orthopaedic conditions requires clinical decision-making in the context of the whole child and not just specific technical surgery. As such it is a much welcomed and strategically important contribution.

This is particularly the case since in recent times there has been almost an explosion of paediatric orthopaedic text books. This book, however, is very different and I predict will be a mandatory reference in the out-patient department mainly for residents and fellows but also as a teaching aid for specific cases. The book focuses on clinical problems within regional pathology and here lies the book's success. Limb deficiencies are grouped together and sensible consensus-based management approaches co-ordinated. Problems are highlighted and all the text augmented as indeed is the case throughout the book by a single colour tone (blue) table and illustrational construct. Limb-length discrepancy similarly addresses all four limbs in a single section. Controversially, perhaps, there is no single chapter on cerebral palsy but a masterful approach to 'paralyses' in general. Here, the ethos of decision making, clinical features and treatment recommendations provide seminal instructional material.

Wherever the reader seeks information, the decision-making process is safe. The account of septic arthritis may not be the most comprehensive but it is a perfect vignette of decision resolution that often causes severe clinical tension, particularly for residents.

Again, the sections are adequately referenced but do not provide a full literature review - the message is in the text and the tables. Senior surgeons will find this book invaluable as a prompt for clinical teaching in the clinic. At present, this text is the most informative and practical summary of paediatric orthopaedics available; as an adjunct to the standard texts, it should be unreservedly recommended. 